\title{
Eligibility for medical thromboprophylaxis based on risk-factor weights, and clinical thrombotic event rates
} \section{J Alasdair Millar
PhD, FRACP. FRCP Physician and Clinica Pharmacologist? and Physician and Clinical Director $^{2}$ \\ Joanne E Lett $\mathrm{MB}$ ChB Registrar, Medicine Department \\ Leonard J Bagley BPharm Hospital Pharmacist ${ }^{2}$ \\ Ian K Densie \\ Southland Medica Foundation Research Officer $^{2}$}

1 Albany Regional Hospital, Albany, WA.

2 Southland Hospital, Invercargill, New Zealand.

alasdair.millar@ health.wa.gov.au

MJA 2012; 196: 457-461 doi: 10.5694/mjall.10737

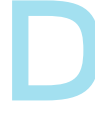

vein thrombosis (DVT) and pulmonary embolism (PE) arising in medical inpatients may be preventable using anticoagulant prophylaxis. Guidelines for thromboprophylaxis exist, ${ }^{1-3}$ but are problematic in some respects. For example, the Australia and New Zealand Working Party guidelines ${ }^{1}$ (WPG) and American College of Chest Physicians $^{3}$ guidelines (ACCPG) quote VTE incidence as $17 \%$ and $10 \%-20 \%$ of admissions, respectively, because of reliance on clinical trials where the diagnosis was of subclinical events. The National Health and Medical Research Council clinical practice guidelines (NHMRCG) ${ }^{2}$ do not report incidence data. The incidence of clinical venous thromboembolism (VTE) based on morbidity coding data ranges from $0.4 \%$ to $0.67 \%$. ${ }^{4-6}$ The Australasian guidelines ${ }^{1,2}$ are based on the presence of risk-factors without taking into account their individual statistical weights. We have previously argued for the use of patient selection criteria to prevent clinical VTE events, based on improving the benefit-to-hazard ratio of thromboprophylaxis by taking into account relative risk-factor weighting. ${ }^{7}$

We aimed to develop a practical scheme of eligibility for medical VTE prophylaxis according to the statistical weights of known risk factors, ${ }^{8,9}$ and to compare the eligibility of patients under this scheme with eligibility under existing guidelines. We also aimed to measure prospectively the incidence of clinical thromboembolism in medical inpatients. Based on this work, we propose new eligibility criteria for medical thromboprophylaxis that are consistent with the NHMRCG. ${ }^{2}$

\section{Methods}

The study was a prospective hospital case-note audit and did not require ethics committee approval or patient consent. ${ }^{10}$ Results of a pilot study in 52 patients have been presented

Objectives: To measure eligibility for medical thromboprophylaxis using two Australasian guidelines - the Australia and New Zealand Working Party Guidelines [WPG] and the National Health and Medical Research Council Guidelines [NHMRCG]) - and proposed new guidelines based on risk-factor weights; and to measure the incidence of clinical venous thromboembolism (VTE) events in medical patients ("ensuing VTE").

Design, setting and patients: Prospective case-note audit in an acute medical ward of Southland Hospital, a regional hospital in Invercargill, New Zealand, among all 595 patients who were discharged consecutively from 21 November 2010 to 7 March 2011. Of these, 245 were excluded on clinical grounds or because they were under the care of the authors.

Main outcome measures: The primary outcome was eligibility for prophylaxis under each guideline. Secondary outcomes included incidence of ensuing VTEs, use of thromboprophylaxis, drug acquisition costs with each guideline, and bedside practicability of a guideline based on risk-factor weights.

Results: Nineteen per cent of patients were eligible under the new guidelines, compared with $80 \%, 88 \%$ and $36 \%$ under the WPG and two interpetations of the NHMRCG, respectively. One patient had an ensuing VTE. The new guideline had lower drug acquisition costs and was suitable for bedside use.

Conclusions: Clinical VTE events are rare in medical patients, and medical VTE thromboprophylaxis needs to be more focused. The new guideline has performance characteristics that satisfy this need.

previously. ${ }^{11}$ The primary outcome was eligibility for prophylaxis, measured according to four sets of criteria from three guidelines, taking bleeding risk into account.

Set 1: $\mathbf{W P G}^{1}$ - These guidelines promote thromboprophylaxis in patients at "high" and "low" risk based on the presence of any one of seven factors (age > 60 years, stroke, history of VTE, cancer, heart failure, acute on chronic lung or inflammatory disease) without regard to factor weight.

Sets 2 and 3: NHMRCG - These guidelines promote "consideration" of thromboprophylaxis according to risk assessment but do not provide explicit rules for eligibility in individuals. We applied the guidelines either broadly - assigning eligibility if any risk factor was present, unless contraindicated by any bleeding risk (NHMRCG1) - or, more conservatively - as published in the New South Wales Department of Health policy document, Appendix 2 (NHMRCG2). ${ }^{12}$

Set 4: New risk-based guidelines (RBG) - Empirical guidelines devised according to the relative risk weighting of known VTE risk factors, ${ }^{8,9}$ stratified into three groups of decreasing risk. These criteria (Box 1) were intended to exclude patients whose VTE risk was less than that of prophylaxis-induced major bleeding, ${ }^{7}$ while retaining consistency with the NHMRCG, and with simplicity of bedside application. The criteria were amended on the basis of the results of the pilot study ${ }^{11}$ and further modified in the light of clinical experience after the first 60 patients had been studied. Data collected to that point were unaffected by the adjustments.

We examined the case records of all medical patients who died or were discharged from Southland Hospital, Invercargill, New Zealand, from 21 November 2010 to 7 March 2011. Patients were excluded either for medical reasons (use of anticoagulant therapy at or prescribed during admission for reasons other than DVT or PE prophylaxis; admission to the intensive care or rehabilitation units; admission for palliation; being co-managed with another specialty; transfer to another 
hospital within 2 days of admission; day admission; or to avoid bias [patients admitted under or managed by the authors]). By definition, patients with an admission diagnosis of DVT or PE were excluded.

Patients were identified as having ensuing VTE from records in the clinical notes of any VTE diagnosis during admission; any indication of lowerlimb swelling or tenderness, chest pain, dyspnoea, or haemoptysis; use of treatment-dose enoxaparin $(1 \mathrm{mg} / \mathrm{kg}$ twice daily, or $1.5 \mathrm{mg} / \mathrm{kg}$ daily) not due to other indications; or readmission with a primary VTE diagnosis up to 90 days ${ }^{5}$ after discharge.

We continued examining case records until the cumulative data reached a steady state for the proportion of patients eligible for prophylaxis under the WPG $( \pm 1 \%$ over three data points at intervals of 25 admissions), plus an additional 50 admissions to ensure data maturity. We collected demographic and clinical information, and data on clinical symptoms on admission, underlying medical diagnoses, occurrence of ensuing VTE, and enoxaparin administration (at a dose of $40 \mathrm{mg}$ or $20 \mathrm{mg}$ daily, depending on renal function above or below $30 \mathrm{~mL} /$ $\min / 1.73 \mathrm{~m}^{2}$ by eGFR, respectively). ${ }^{13}$ We assessed eligibility for thromboprophylaxis under each guideline set. We estimated annual costs in Australia under each guideline at full compliance using inpatient data published by the Australian Institute of Health and Welfare. ${ }^{14}$ We did not intervene in the management of any patient or analyse data by treating physician. Results were presented only after the study ended.

We checked the audit's internal validity by measuring ensuing VTE using hospital discharge coding data, based on ICD-10 (International Classification of Diseases, 10th revision) classifications. We examined the records of patients who died during admission to assess whether PE could be a likely cause of death on clinical grounds (no autopsies were performed). Data on deaths in the community up to 90 days after admission were not available.

\section{Results}

Study end points reached steady state after 300 patients were included, but

1 Risk-based criteria included in the new guidelines

Level 1

- Active cancer

- Previous history of DVT or PE

- Untreated thrombophilia

Level 2

- Post-phlebitic syndrome

- Surgical procedure requiring a general anaesthetic or ICU admission in previous 30 days

- Multiorgan sepsis with failure of at least one organ

Level 3

- Severe peripheral vascular disease with threat of amputation

- Grade IV heart failure

- Symptomatic lung disease sufficiently severe to require BPAP or $\mathrm{O}_{2}$ therapy

- Non-stroke neurological disease with paresis and immobility

- Acute colitis or other severe diarrhoeal disease or severe inflammation or UTI with dehydration and CRP $>200$

Eligibility

Patients were eligible for thromboprophylaxis with the following combinations of the above levels:

- any from Level 1

- any two from Level 2

- any three from Level 3

- any one from Level 2 plus any two from Level 3

DVT = deep vein thrombosis. $P E=$ pulmonary embolism. ICU = intensive care unit. BPAP = bilevel positive airway pressure. $\mathrm{UTI}=$ urinary tract infection. $\mathrm{CRP}=\mathrm{C}$-reactive protein.

\section{Reasons for exclusion of patients from the study}

Reason

No.

Involvement of the authors in the medical care of the patient

2 Patient taking anticoagulant on admission or therapeutic anticoagulation* started during admission

3 Combination of 1 and 2

86

10

4 Transferred to or from another department or hospital

5 Intensive care unit admissions

6 Procedures (day-only admissions to general ward)

7 Other

Total

Therapeutic anticoagulation did not include patients requiring anticoagulants for ensuing deep vein thrombosis or pulmonary embolism.

this was noted to be within a slow downward trend that continued after addition of a further 50 patients. Of 595 patients screened, 245 (41.2\%) were excluded (350 included). Reasons for exclusion are shown in Box 2 . Among 287 patients who were not given prophylaxis, one case of ensuing VTE was observed (upper 95\% confidence limit, 5.6 cases) - a PE (possibly present on admission) discovered on staging computed tomography in an 89-year-old woman with metastatic malignancy of the caecum.

Eligibility under the guidelines is shown in Box 3. The highest eligibility was with NHMRCG1 (88.0\%), attributable mainly to the criterion "age $>40$ years". The lowest eligibility was found with the RBG (19.1\%), dominated by the factor "active cancer".
Enoxaparin was the only drug used for thromboprophylaxis. Physical prophylaxis was not used except in very occasional patients given compression stockings by nursing staff (not counted as prophylaxis for study purposes). The proportion of inpatients given prophylactic enoxaparin was $18.0 \%$ overall (63/350), but without any discernible pattern. Of the 294 patients eligible under the WPG, 237 (80.6\%) were not given prophylaxis, compared with 49 of the $72(68.1 \%)$ who were eligible under the RBG. Of the patients who were not eligible under each of these two guidelines, respectively, $10.7 \%(6 / 56)$ and $14.7 \%(41 / 278)$ were given prophylaxis.

The morbidity coding check supported the accuracy of the study 
documentation, but revealed two further patients with VTE on admission. One had a superficial phlebitis at an upper limb cannula site. The second was missed, but the PE coding was historical and the patient was taking warfarin. Ten study patients died during admission. All deaths were expected and readily explicable on the basis of the primary diagnosis (details available from the authors) and were considered unlikely to have been due to a VTE event.

The estimated annual national drug costs of treating Australian patients who would be eligible to receive enoxaparin under each guideline, assuming full compliance, are shown in Box 4. The annual potential saving with use of the RBG under these conditions is $\$ 29.0$ million, $\$ 6.6$ million and \$25.6 million compared with using NHMRCG1, NHMRCG2 and WPG, respectively.

Some prescribing errors were noted in the course of the study. Thirteen patients with eGFR $>30 \mathrm{~mL} / \mathrm{min} /$ $1.73 \mathrm{~m}^{2}$ were given enoxaparin $20 \mathrm{mg}$ daily. Conversely, two patients with
eGFR $<30 \mathrm{~mL} / \mathrm{min} / 1.73 \mathrm{~m}^{2}$ received $40 \mathrm{mg}$ daily. One patient was given enoxaparin at a therapeutic dose (100 mg daily) when the stated indication was prophylaxis.

\section{Discussion}

We have confirmed by direct observation that ensuing clinical VTE events in acute medical patients are rare, and consistent with published incidence ${ }^{4-6}$ of about $0.5 \%$. On this basis, we expected to see one or two cases in the 287 patients not given prophylaxis, and the one patient with an ensuing $\mathrm{PE}$ was consistent with this. The reported VTE incidence relied on by the WPG predicts 48 cases. While acknowledging that their data include non-clinical events, the guidelines do not state the proportion. We argue that there is no case for preventing an asymptomatic condition unless it can be shown that a high proportion of cases progress to symptomatic disease. No studies have addressed this possibility directly, but even symptomatic distal thromboses do not usually

3 Overall gross (without bleeding risk exclusions) and net eligibility (\%) for thromboprophylaxis in $\mathbf{3 5 0}$ general medical inpatients, according to the guideline sets

\begin{tabular}{lcccc} 
Guideline & Eligibility (gross) & Eligibility (net) & $\begin{array}{c}\text { Dominant risk } \\
\text { factor(s)* }\end{array}$ & Contribution $^{\dagger}$ \\
\hline NHMRCG1 & $313(89.4 \%)$ & $308(88.0 \%)$ & Age $>40$ years & $97.1 \%$ \\
WPG & $294(84.0 \%)$ & $280(80.0 \%)$ & Age $>60$ years & $88.8 \%$ \\
NHMRCG2 & $131(37.4 \%)$ & $126(36.0 \%)$ & $\begin{array}{c}\text { Chest infection, heart } \\
\text { failure, cancer }\end{array}$ & $93.1 \%$ \\
RBG & $72(20.6 \%)$ & $67(19.1 \%)$ & Active cancer & $86.1 \%$ \\
\hline
\end{tabular}

NHMRCG $=$ National Health and Medical Research Council guidelines. WPG $=$ Australia and New Zealand Working Party Guidelines. RBG = risk-based guidelines. * Dominant eligibility criterion. † Contribution, alone or in combination with at least one other risk factor, to the overall gross eligibility. progress to proximal disease. ${ }^{15}$ As Invercargill has only one public hospital, cases are unlikely to have been missed due to readmission to another hospital. However, VTE events may have occurred in patients who died after discharge. Excluding this qualification, our data suggest that even patients who are at high risk of DVT, and are known from clinical trials to develop asymptomatic VTE in nearly one-fifth of cases, manifest clinical disease in relation to a hospital admission at a rate of about 1 in 287 $(0.35 \%)$.

This low incidence might be taken to indicate that thromboprophylaxis is not required in acutely unwell patients admitted to a medical ward. However, potentially fatal but preventable VTE events do occasionally occur, particularly in patients who have easily identified risk factors of substantial weight. This is the case for applying weight-based guidelines for prophylaxis.

We have shown that the NHMRCG1 and WPG allocate 88\% and $80 \%$ of medical inpatients to prophylaxis, respectively, thus providing almost universal partial protection (the trial-based relative risk reduction is about $0.5^{16}$ ) against a VTE event, with a number needed to treat of up to $400^{16}$ and significant drug-acquisition costs. The WPG effectively ignore the bleeding risk of prophylaxis, but this factor is recognised by the NHMRCG. In contrast, our guideline focuses on patients at a higher level of thrombotic risk, leading to a more targeted risk reduction, thereby improving the benefit-to-hazard ratio and decreasing

\section{National costs and potential savings (Australia) at 100\% compliance with the NHMRCG1, NHMRCG2, WPG and RBG}

\begin{tabular}{|c|c|c|c|c|c|c|}
\hline \multirow[b]{2}{*}{ Row } & \multirow[b]{2}{*}{ Item } & \multicolumn{4}{|c|}{ Value } & \multirow[b]{2}{*}{ Source } \\
\hline & & NHMRCGI & NHMRCG2 & WPG & RBG & \\
\hline 1 & Overnight admissions & \multicolumn{4}{|c|}{2362371} & $\operatorname{AlHW}^{14}$ (s.7, table 9.2) \\
\hline 2 & Age $<20$ years $(\%)$ & \multicolumn{4}{|c|}{$9.48 \%$} & $\operatorname{AlHW}^{14}(\mathrm{~s} .9$, table 7.4$)$ \\
\hline 3 & Adult admissions & \multicolumn{4}{|c|}{2138418} & $\operatorname{row} 1 \times(1-\operatorname{row} 2)$ \\
\hline 4 & LOS (days) & \multicolumn{4}{|c|}{4.3} & AlHW $^{14}$ (s.7, table 9.14) \\
\hline 5 & Total bed-days & \multicolumn{4}{|c|}{9195198} & row $3 \times$ row 4 \\
\hline 6 & 40 mg enoxaparin ( $\$ /$ day) & \multicolumn{4}{|c|}{$\$ 4.57$} & Cost to pharmacy \\
\hline 7 & Proportion eligible & 0.880 & 0.349 & 0.800 & 0.191 & This study \\
\hline 8 & Bed-days* & 8091774 & 3209124 & 7356158 & 1756283 & row $5 \times$ row 7 \\
\hline 9 & Annual cost, full compliance* & $\$ 36979408$ & $\$ 14665697$ & $\$ 33617644$ & $\$ 8026213$ & row $6 \times$ row 8 \\
\hline 10 & Excess annual cost ${ }^{\dagger}$ & $\$ 28953195$ & $\$ 6639484$ & $\$ 25591431$ & - & Non-RBG-RBG, row 9 \\
\hline
\end{tabular}

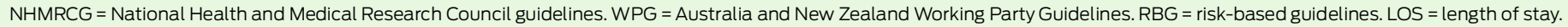
AlHW = Australian Institute of Health and Welfare. * Values shown according to each guideline. $†$ Excess cost compared with RBG. 
drug costs. In our study, NHMRCG2 provided an intermediate eligibility and appeared to be a more satisfactory interpretation of the NHMRC guidelines. Our guidelines are consistent with NHMRCG, which state

... individual assessment of the

VTE risk is recommended for each patient,

and,

Depending on their magnitude the risk factors related to an injury, a surgical procedure, or an acute medical illness often exert a dominating influence for their duration. ${ }^{2}$

Our guidelines were developed empirically and lack formal validation, but this applies to all thromboprophylaxis guidelines, including the WPG, ${ }^{1}$ NHMRCG and ACCPG. ${ }^{3}$ A similar approach to ours, based on allocation of points for individual risk factors, was described by Chopard et al, ${ }^{17}$ who also acknowledged lack of validation. In fact, no procedures exist to validate any guideline in an epistemological sense. It might be claimed that the validation for the guidelines lies in the clinical trials showing benefit, but in the case of thromboprophylaxis the trials ${ }^{18-20}$ measured subclinical events detected by imaging techniques and thus provide only circumstantial evidence that prophylaxis decreases clinical event rates. One of these studies ${ }^{20}$ and a meta-analysis ${ }^{16}$ suggest that any reduction is small.

The rate of medical thromboprophylaxis use at Southland Hospital is low, compared with the eligibility conferred by the WPG and in agreement with various studies of guideline compliance. ${ }^{21-24}$ Such studies do not contain data on VTE rates but invariably lament the "low" use of prophylaxis and argue for improved compliance. They do not question the underlying validity of the guidelines. Though thromboprophylaxis use in our study was of the same order suggested by our proposed guidelines, this was coincidental. Use was arbitrary and, for that reason, unsatisfactory.

We have shown that it is possible to allocate prophylaxis to general medical patients in a hierarchical fashion according to risk-factor weights, of which the strongest are active cancer (with and without chemotherapy), and previous history of DVT. ${ }^{8,9}$ Though we made provision for risk factors of lesser weight, they did not contribute substantially to the overall result. It may therefore be appropriate to provide medical thromboprophylaxis only on the basis of active cancer, previous history of DVT or PE, and known but untreated thrombophilia. Such a policy would have identified $92 \%$ of our eligible patients. We considered the desirability of including a criterion of severe but reversible debility of any cause, including rare but severe conditions with no published risk weight. Again, this would not contribute substantially to the overall result.

In our view, the WPG and NHMRCG1 are flawed because the eligibility they confer is driven predominantly by age, a statistically significant but relatively weak risk factor for thrombosis. Age is also a risk factor for bleeding during anticoagulant therapy when used for thromboprophylaxis in atrial fibrillation ${ }^{25}$ and after myocardial infarction ${ }^{26}$ or PE. ${ }^{27}$ The cut-off age of 60 years in the WPG is not evidence-based because the supporting clinical trials were in patients aged over 40 years, as reflected in the NHMRCG. The effect of these age criteria is to confer eligibility in a substantial majority of the acute medical inpatient population ${ }^{24}$ for a clinical condition whose average absolute risk, described in the WPG as "high", must in fact be close to the population average.

By contrast, our proposed riskbased guidelines assign eligibility based on risk-factor weights with relative risk $\geqslant 5,{ }^{8,9}$ and confer eligibility in about $20 \%$ of the patient population. Their use would also reduce drug-acquisition costs substantially. The overall cost-effectiveness remains uncertain, as this would depend on the relative number of VTEs that occurred with each set of guidelines in use, the cost of treating each VTE and their sequelae, and the cost of treating haemorrhagic complications. Nevertheless, the results of this study reinforce our earlier concerns over the genesis $^{28}$ and content ${ }^{29}$ of the WPG and render a broad interpretation of the NHMRCG, such as NHMRCG1, unattractive. Similar concerns have recently been expressed in the United Kingdom $^{30,31}$ and United States. ${ }^{32,33}$

Our study has several limitations. First, the guideline comparisons are of eligibility, not efficacy. Measuring the comparative effect on prevention of clinical VTE events would require a very large clinical trial. However, guidelines are not validated by comparative studies; rather, they arise from an expert distillation of current knowledge and are validated by their underlying logic and professional acceptance. Second, our study may have suffered from ascertainment bias due to missed VTE events during casenote review in spite of observer skill, or missed events in patients who died during admission or after discharge. The latter cases can be ascertained only by autopsy. Our checks using morbidity coding suggest that these factors were small in magnitude, although hospital coding has limitations when used for research.

In summary, we have provided evidence of the rarity of symptomatic medical ensuing VTE and of the practicability of guidelines based on relative risk-factor weight that are consistent with the NHMRCG. We propose that this approach be considered for routine application for medical thromboprophylaxis.

Acknowledgements: We thank the Southland Medical Foundation for their support of the Southland Medical Foundation Research Officer (Ian Denise).

Competing interests: No relevant disclosures.

Received 13 Jun 2011, accepted 13 Feb 2012.

1 The Australia and New Zealand Working Party on the Management and Prevention of Venous Thromboembolism. Prevention of venous thromboembolism. Best practice guidelines for Australia and New Zealand. 4th ed. Health Education \& Management Innovations, 2007. http://www.vascular.co.nz/ANZ\%20VTE Guidelines.pdf (accessed Mar 2012).

2 National Health and Medical Research Council. 2009 Clinical practice guideline for the prevention of venous thromboembolism (deep vein thrombosis and pulmonary embolism) in patients admitted to Australian hospitals. Melbourne: NHMRC, 2009. http:// www.nhmrc.gov.au/nics/programs/vtp/ prevention.htm (accessed Jan 2011).

3 Geerts WH, Bergqvist D, Pineo GF, et al. Prevention of venous thromboembolism: American College of Chest Physicians evidencebased clinical practice guidelines (8th ed). Chest 2008; 133 (6 Suppl): 381S-453S.

4 Millar JA, Lee GE, lenco R. Prevalence of venous thromboembolism in medical inpatients [letter]. Med J Aust 2010; 192: 724-726.

5 National Institute of Clinical Studies. The incidence and risk factors for venous thromboembolism in hospitals in Western Australian 1999-2001. Melbourne: NICS, 2005. http://www.nhmrc.gov.au/nics/material_ 
resources/resources/incident_risk.htm (accessed Mar 2012)

6 Montero Ruiz E, Baldominos Utrilla G, López Álvarez J, Santolaya Perrin R. Effectiveness and safety of thromboprophylaxis with enoxaparin in medical patients. Thromb Res 2011; 128: 440445. [Epub ahead of print 30 Aug 2010.]

7 Millar JA. Selection of medical patients fo prophylaxis of venous thromboembolism based on analysis of the benefit-hazard ratio. Intern Med J 2009; 39: 606-612.

8 Edelsberg J, Hagiwira M, Taneja C, Oster G. Risk of venous thromboembolism among hospitalized medically ill patients. Am J Health Syst Pharm 2006; 63 (20 Suppl 6): S16-S22.

9 Alikhan R, Cohen AT, Combe S, et al; MEDENOX Study. Risk factors for venous thromboembolism in hospitalized patients with acute medical illness: analysis of the MEDENOX Study. Arch Intern Med 2004; 164: 963-968.

10 New Zealand National Ethics Advisory Committee. Ethical guidelines for observational studies: observational research, audits and related activities. Wellington: Ministry of Health, 2006. http://www.neac.health.govt.nz/moh.nsf/ pagescm/520/\$File/ethicalguidelines.pdf (accessed Jan 2011).

11 Millar JA, Wright CA. Guidelines for medical thromboprophylaxis based on risk factor weights: pilot study. Abstract 2.6. Proceedings of the Australasian Society of Clinical and Experimental Pharmacologists and Toxicologists; 2010 Aug 2931. Christchurch, New Zealand.

12 NSW Department of Health Clinical Safety, Quality and Governance Branch. Prevention of venous thromboembolism. Policy directive, document no. PD2010_077. Sydney: NSW Health, 2010. http://www.health.nsw.gov.au/policies/ pd/2010/pdf/PD2010_077.pdf (accessed Sep 2011)

13 Levey AS, Bosch JP, Lewis JB, et al. A more accurate method to estimate glomerular filtration rate from serum creatinine: a new prediction equation. Modification of Diet in Renal Disease Study Group. Ann Intern Med 1999; 130: 461-470.

14 Australian Institute of Health and Welfare. Australian hospital statistics 2009-10. Canberra:
AlHW, 2011. (AlHW Cat. No. HSE 107; Health Services Series No. 40.) http://www.aihw.gov.au/ publication-detail/?id=10737418863\&tab=2 (accessed Jun 2011)

15 Singh K, Yakoub D, Giangola P, et al. Early followup and treatment recommendations for isolated calf deep vein thrombosis. J Vasc Surg 2012; 55: 136-140. [Epub ahead of print 7 Oct 2011.] http:// www.jvascsurg.org/article/S0741-5214(11) 01849-0/abstract (accessed Nov 2011).

16 Dentali F, Douketis JD, Gianni M, et al. Metaanalysis: anticoagulant prophylaxis to prevent symptomatic venous thromboembolism in hospitalized medical patients. Ann Intern Med 2007; 146: 278-288.

17 Chopard P, Spirk D, Bounameaux H. Identifying acutely ill medical patients requiring thromboprophylaxis. J Thromb Haemost 2006; 4 915-916.

18 Cohen AT, Davidson BL, Gallus AS, et al; ARTEMIS Investigators. Efficacy and safety of fondaparinux for the prevention of venous thromboembolism in older acute medical patients: randomised placebo controlled trial. BMJ 2006; 332: 325-329.

19 Samama MM, Cohen AT, Darmon JY, et al. A comparison of enoxaparin with placebo for the prevention of venous thromboembolism in acutely ill medical patients. Prophylaxis in Medical Patients with Enoxaparin study Group. NEngl J Med 1999; 341: 793-800.

20 Leizorovicz A, Cohen AT, Turpie AG, et al; PREVENT Medical Thromboprophylaxis Study Group. Randomized, placebo-controlled trial of dalteparin for the prevention of venous thromboembolism in acutely ill medical patients. Circulation 2004; 110: 874-879.

21 Cohen AT, Tapson VF, Bergmann JF, et al; ENDORSE Investigators. Venous thromboembolism risk and prophylaxis in the acute hospital care setting (ENDORSE study): a multinational cross-sectional study. Lancet 2008; 371: 387-394.

22 Burleigh E, Wang C, Foster D, et al. Thromboprophylaxis in medically ill patients at risk for venous thromboembolism. Am J Health Syst Pharm 2006; 63 (20 Suppl 6): S23-S29.
23 Amin A, Stemkowski S, Lin J, Yang G. Thromboprophylaxis rates in US medical centers: success or failure? J Thromb Haemost 2007; 5 : 1610-1616.

24 Gibbs H, Fletcher J, Blombery P, et al. Venous thromboembolism prophylaxis guideline implementation is improved by nurse directed feedback and audit. Thromb J 2011:9: 7. http:// www.thrombosisjournal.com/content/9/1/7 (accessed Mar 2012)

25 Hughes M, Lip GY. Risk factors for anticoagulation-related bleeding complications in patients with atrial fibrillation: a systematic review. OJM 2007; 100: 599-607.

26 Kadakia MB, Desai NR, Alexander KP, et al; National Cardiovascular Data Registry. Use of anticoagulant agents and risk of bleeding among patients admitted with myocardial infarction: a report from the NCDR ACTION Registry--GWTG. JACC Cardiovasc Interv 2010; 3: 1166-1177.

27 Mikkola KM, Patel SR, Parker JA, et al. Increasing age is a major risk factor for haemorrhagic complications after pulmonary embolism thrombolysis. Am Heart J 1997; 134: 69-72.

28 Millar JA. Genesis of medical thromboprophylaxis guidelines in Australia: a need for transparency and standardisation in guideline development. Med J Aust 2009; 190: 446-450.

29 Millar JA. Rational thromboprophylaxis in medical inpatients: not quite there yet. Med J Aust 2008; 189; 504-506.

30 Welfare M. NICE's recommendations for thromboembolism are not evidence based. BMJ 2011; 343: d6452.

31 D'Costa DF. Time to reconsider NICE guidance on heparin prophylaxis in medical inpatients. BMJ 2011; 343: d7886.

32 Lederle FA, Zylla D, MacDonald R, Wilt TJ. Venous thromboembolism prophylaxis in hospitalized medical patients and those with stroke: a background review for an American College of Physicians Clinical Practice Guideline. Ann Intern Med 2011; 155: 602-615.

33 Allareddy R. Skeptical about the sweeping recommendations. Ann Intern Med online forum 2011; 7 Nov. http://www.annals.org/content/155/ 9/602.abstract/reply\#annintmed_el_127522 (accessed Jan 2012). 